\title{
The relation between task-relatedness of anxiety and metacognitive performance
}

Catherine Culot ${ }^{1}$, Gaia Corlazzoli ${ }^{1}$, Carole Fantini-Hauwel ${ }^{2} \&$ Wim Gevers $^{1}$

1. Center for Research in Cognition and Neurosciences (CRCN) - Université Libre de Bruxelles (ULB), Brussels, Belgium

Neurosciences Institute (UNI), Université Libre de Bruxelles (ULB), Brussels,

$$
\text { Belgium }
$$

2. Research center for experiMEntAl \& clinical cogNitive psychopatholoGy (MEANING), Université Libre de Bruxelles (ULB), Brussels, Belgium

\section{Corresponding author:}

Catherine Culot

Center for Research in Cognition and Neurosciences - Université Libre de Bruxelles

Av. F. Roosevelt 50/CP191 - 1050 Brussels - Belgium

Tel: +3226502631

cculot@ulb.ac.be 


\begin{abstract}
It is well established that anxiety influences a range of cognitive processes such as cognitive control or decision-making. What is less known is how anxiety influences the metacognitive evaluations individuals make about their own performance. The present study explored the importance of task-relatedness in the relation between anxiety and metacognitive awareness. More specifically, we hypothesized that induced anxiety would affect metacognitive performance in opposite directions depending solely on whether the threat is perceived as related or unrelated to the performance. We conducted two experiments to test this hypothesis. In both studies, electric shocks were administered randomly, regardless of participant's performance. In experiment 1, participants were informed that the shocks would be administered randomly, unrelated to performance. In experiment 2, participants were told that their metacognitive performance would influence the administration of the electric shocks (i.e. fewer electric shocks with better metacognitive performance). Our predictions were confirmed. Threat perceived as unrelated to metacognitive performance (Experiment 1) decreased metacognitive performance. Threat believed to be related to metacognitive performance resulted in improved performance (Experiment 2).

These findings confirm that induced anxiety exerts a strong influence on metacognitive awareness while sparing first-order task performance. We furthermore demonstrate that the perceived relatedness of the anxiety determines whether metacognitive performance decreases or improves performance under threat.
\end{abstract}

Keywords: Anxiety; Task-relatedness; Confidence; Metacognition; Threat of shock

\title{
Highlights
}

- The nature of the task and the perceived task-relatedness of anxiety are two factors contributing to the relation between anxiety and cognition.

- High-order (e.g. metacognitive) processes are affected by anxiety while low-order (e.g. perceptual) performance is not.

- Metacognitive performance improves when the threat is administered randomly but perceived as directly related to metacognitive performance. 
- Metacognitive performance decreases when the threat is perceived as unrelated to metacognitive performance.

\section{Introduction}

Anxiety is an emotional state characterized by worrisome thoughts about uncertain negative events that is adaptive in nature but can become pervasive and detrimental in some cases (Moser, Moran, Schroder, Donnellan, \& Yeung, 2013). An extensive body of literature has demonstrated that anxiety, as many emotions, exerts a strong influence on a range of cognitive processes such as working memory or decision-making (see Robinson, Vytal, Cornwell, \& Grillon, 2013 for a review). However, the direction (positive or negative) of this influence varies from one situation to another. For instance, stress and anxiety can boost the processing of threat related stimuli but can also become detrimental when the cognitive task becomes more demanding and requires higher order cognitive functions (Robinson et al., 2013). A better understanding of the factors determining whether anxiety will increase, will decrease or will have no impact on performance is therefore crucial.

As with many emotions, anxiety is thought to act on cognitive performance mainly through its impact on attentional processes (Pourtois, Schettino, \& Vuilleumier, 2013; Siman-Tov et al., 2009). Threat-related stimuli are particularly salient and capture attention in a bottom up manner, which prioritizes their processing. This "emotional salience" is viewed as an evolutionary process enabling a rapid detection of important stimuli in the environment like a potential danger (Anderson \& Phelps, 2001; Iordan, Dolcos, \& Dolcos, 2013; Paradiso, 1998). Emotional salience relates to the notion of resource competition that was developed in the Processing Efficiency Theory (Eysenck, Derakshan, Santos, \& Calvo, 2007). Worries and threat are salient features that compete with the task for cognitive resources. This increases the amount of effort needed to perform the task correctly. In cases of relatively easy tasks, anxiety would not lower performance because participants can easily recruit additional cognitive resources (Hadwin, Brogan, \& Stevenson, 2005; Smith, Bellamy, Collins, \& Newell, 2001). Performance would suffer only if additional resources cannot be recruited, as is the case in more demanding tasks or dual task paradigms (Murray \& Janelle, 2003). Likewise, some clinical studies reported that anxiety disorders had more impact on tasks requiring higher levels of cognitive control compared to easier, more automatized, tasks (Ashcraft \& Kirk, 2001; Wood, Mathews, \& Dalgleish, 2001). Following this idea, high-order cognitive functions would be more influenced by anxiety than low-order processes. 
The differentiated impact of anxiety on low vs high-order cognitive processes can nicely be studied in perceptual metacognitive tasks. Metacognition is a high-order process as it reflects the ability to think about and evaluate our own cognition and performance (Flavell, 1979; Fleming, Ryu, Golfinos, \& Blackmon, 2014). Perceptual metacognitive tasks require participants to first perform a perceptual decision (low-order task) and then to rate their confidence in their performance (high-order metacognitive judgement). Interestingly, even though combined within a single task, low and high-order cognitive processes have been shown to operate independently of one another (Palmer, David, \& Fleming, 2014; Fleming et al., 2014; Rounis, Maniscalco, Rothwell, Passingham, \& Lau, 2010). That is, good objective perceptual performance does not necessarily imply good metacognitive accuracy, and vice versa. Using this approach, one study measured stress responsiveness as a personality trait (via measures of cortisol concentrations one year before the testing) (Reyes, Silva, Jaramillo, Rehbein, \& Sackur, 2015). Participants were divided in three groups (high, medium and low stress) according to this measure. All participants performed a perceptual metacognitive task. Equal performance was observed across the different groups on the low-order visual discrimination task. However, high-order metacognitive performance (the ability to discriminate correct from incorrect performance) was impaired in high stress responders. A similar perceptual metacognitive study was conducted by Massoni (2014). This time, worrying ${ }^{1}$ was induced during the task by associating financial rewards or penalties to the accuracy of the metacognitive judgments. As in the study of Reyes and colleagues (2015) first-order perceptual performance was not affected by worrying while metacognitive performance was. Surprisingly however, worrying improved rather than decreased metacognitive performance.

Both studies hold in common that first-order perceptual processing was not influenced by emotion while this was the case for high-order metacognitive judgments. In line with the Processing Efficiency Theory one could assume that the relatively simple nature of the perceptual task left sufficient capacity to deal with the emotional disposition (Reyes et al., 2015) or the emotional induction (Massoni, 2014). However, it seems insufficient to explain the direction of this impact. Why did metacognitive performance decrease in the study of Reyes and colleagues (2015) but improved in the study of Massoni (2014)?

We make the hypothesis that a second yet unexplored factor may have played an important role in the direction of this relation: task-relatedness of the induced emotion. A possible crucial

\footnotetext{
${ }^{1}$ Note that Massoni (2014) used the term worry instead of anxiety to define the emotional state induced by their manipulation. However, they defined worry as "being accompanied by feeling of anxiety" and explicitly indicated that they use worry as a proxy for anxiety.
} 
difference between both studies (e.g. Massoni, 2014; Reyes et al., 2015) was the relatedness of the emotion for task performance. In the study of Massoni (2014), the worrying state was taskrelated: participants could lower the level of threat or worrying by improving their metacognitive performance. Instead, in the study of Reyes et al (2015), emotion was taskunrelated: stress was measured as a general disposition and could not be changed by task performance. With task-unrelated emotions, perceptual performance again remained intact but metacognitive performance decreased. Task-relatedness of the emotion was however not the only difference between both studies. Massoni (2014) induced a worrying state while Reyes and collaborators (2015) measured a trait-like disposition to stress. This could have important implications. Induced anxiety aims to induce an acute anxious state while participants with high disposition to stress were not necessarily in an elevated emotional state at the precise moment of the metacognitive task. Several studies found differences between dispositional and induced anxiety in relation to cognitive processes (see Robinson et al., 2013).

The aim of the present study is to investigate whether task-relatedness is, in itself, an important factor contributing to the relation between anxiety and cognition. We used the same perceptual metacognitive task as Massoni (2014) but instead of using financial reward or penalties we decided to induce anxiety via electric shocks administration. Threat of shocks is the most robust, well-validated induction technique that allows to compare performance under anxious anticipation of rare events (shocks) and performance in a "safe", no-shocks, condition (Davis, Walker, Miles, \& Grillon, 2010; Robinson et al., 2013; Schmitz \& Grillon, 2012; Torrisi et al., 2016). We conducted two experiments in which we manipulated the task-relatedness of the anxiety. Both experiments were identical in set-up. The only difference was in the instruction given to the participants. In both studies, electric shocks were administered randomly, regardless of participant's first- or second-order performance. In experiment 1 , participants were informed that shocks would be administered randomly. In experiment 2 , however, even though electric shocks were again administered randomly, participants were informed that the administration of the shocks would be related to their metacognitive performance. Specifically, participants were explained that they were more at risk to receive an electric shock if they failed to match their objective task performance (i.e. perceptual judgements) with their confidence judgements.

Combining the findings of Reyes et al (2015) and Massoni (2014), we did not expect anxiety to impact the first-order task performance (i.e. perceptual judgment). More importantly, we also hypothesized that the induction of anxiety would have an opposite impact on high-order metacognitive performance depending on its task-relatedness. In experiment 1, the occurrence 
of the electric shocks was presented and perceived to be task-unrelated. In this situation, we predicted that anxiety induction would lead to a decrease in metacognitive performance. In experiment 2, electric shocks were again presented randomly but this time participants believed them to be related to their metacognitive performance. In this situation, we predicted that anxiety induction would lead to an increase in metacognitive performance.

\section{Experiment 1}

\subsection{Method}

\subsubsection{Participants}

Twenty-nine right-handed undergraduate students from the Universite Libre de Bruxelles took part in the study and were paid $10 € /$ hour for their participation. All participants gave written informed consent prior to the experiment. The study was approved by the ErasmusULB Faculty Ethics Committee (P2018/346). Past or current psychiatric and/or neurologic disorder and use of psychoactive medication served as exclusion criteria.

\subsubsection{Anxiety induction}

Electric shocks were delivered using a constant current stimulator (Digitimer DS7A) connected to two electrodes placed on the back of subject's right hand. Individual pain threshold was determined prior to the experiment, such that the stimulation would be "unpleasant but not painful". This threshold was determined by increasing stimulation in steps of $1 \mathrm{~mA}$, following the procedure described in Caspar, Christensen, Cleeremans, \& Haggard (2016). The mean stimulation level obtained by this procedure was $1.9 \mathrm{~mA}(\mathrm{SD}=0.7$, average pulse duration: 520 $\mu \mathrm{s})$.

\subsubsection{Metacognitive task}

The task (see Figure 1) was programmed in MATLAB (MathWorks) with Psychtoolbox (Brainard, 1997) using a modified version of the code of Fleming et al. (2014). On each trial, two circles (diameter $5.1^{\circ}$, eccentricities of $\pm 8.9^{\circ}$ ) were displayed on the screen (Figure 1 ), both containing a fixation cross in its center for $1000 \mathrm{~ms}$. Then the crosses disappeared and a variable number of dots (diameter $0.4^{\circ}$ ) appeared in the circles for $700 \mathrm{~ms}$. Participants were asked to indicate which one of the circles contained the more dots by pressing either "a" (left circle) or " $d$ " (right circle) key on the keyboard. In a random position, one of the circles always contained 50 dots. The other circle displayed a variable number (bounded between 1 and 100). 
The difference in number of dots ("contrast") between the two circles was titrated to fix the performance of each participant to a level of $70 \%$ accuracy using a staircase procedure. After each trial, participants had to indicate their confidence level in the decision they just made. For that purpose, a sliding scale was presented on the screen, ranging from 1 (low confidence) to 6 (high confidence). Participants could move the cursor in a continuous way along the scale with arrow keys, so that the cursor could end up at any possible location, even between the grades.

Figure 1

\subsubsection{Procedure}

Before the start of the experiment, all participants completed the Beck Depression Inventory, $2^{\text {nd }}$ Edition (BDI-II; Beck, Ward, Mendelson, Mock, \& Erbaugh, 1961) translated in French (Bourque \& Beaudette, 1982) as a measure of depressive symptoms and the State-Trait Anxiety Inventory (STAI, Spielberger, Gorsush, Lushene, Vagg, \& Jacobs, 1983) as a measure of anxiety as a general disposition (i.e. as a trait, STAI-B) and anxiety at that particular moment (i.e. as a state, STAI-A). The STAI-A form was completed again at the end of the experiment to measure possible increases in anxiety due to the manipulation.

The metacognitive task consisted of two practice blocks and eight experimental blocks of 25 trials. No confidence ratings were given during the practice blocks and feedback was given after each response. These blocks served to familiarize participants with the task and to initialize the staircase procedure to ensure a $70 \%$ correct level in the experimental blocks.

During the task, two conditions were alternated between blocks: during blocks in the 'threat' condition, participants were told that they would randomly receive some electric shocks. Participants were informed that they could not predict the occurrence of a shock, and that it would not be related to their performance. Instructions and stimuli were displayed in red on a black screen to remind participants throughout the block that they were in the 'threat' condition. Two or three shocks were randomly administrated during the block. During blocks in the 'safe' conditions, participants were told that they would not receive any shocks. The 
experimenter turned off the Digitimer and all instructions and stimuli were presented in white on a black screen.

Participants were assigned to four different groups according to the order of the practice blocks ('safe' - 'threat' vs 'threat' - 'safe') and the first experimental block ('safe' vs 'threat'). Two practice blocks were needed to initialize two separate staircase procedures for the two conditions. A 'safe' block always followed a 'threat' block and vice-versa, such that participants would never perform two consecutive blocks in the same condition.

Between blocks, participants had a break lasting approximately one minute during which they were asked to indicate their subjective anxiety level on a scale from one to ten. Then, instructions on the screen indicated the condition of the next block (i.e. threat or safe). During the blocks, tonic skin conductance level was recorded at a sampling rate of $4 \mathrm{~Hz}$ with an Empatica E4 wristband device (Empatica, Milano, Italy) as a physiological measure of arousal. The skin conductance level (SCL) can be seen as one's overall skin conductance and is measured as an average over an extended period of time (Braithwaite, Watson, Robert, \& Mickey, 2013). Data were extracted using the E4 manager, version 2.0.1. Data were low-pass filtered at $0.05 \mathrm{~Hz}$ and SCL components were extracted using a continuous decomposition analysis with Ledalab (3.4.9, www.ledalab.de) on Matlab (R2015a).

\subsubsection{Data analysis}

Accuracy was defined as percentage of correct responses. Difficulty level was computed as the mean difference in number of dots ('contrast') needed to reach a $70 \%$ accuracy level. The use of confidence ratings after each trial enables to calculate two aspects of metacognition: metacognitive efficiency and metacognitive bias $^{2}$ (Maniscalco \& Lau, 2012). Metacognitive efficiency can be expressed as the mapping between the objective performance and the subjective confidence in the accuracy of such performance (Fleming \& Dolan, 2012). Thus, an individual with high metacognitive efficiency is able to accurately recognize and report fluctuations in his performance (i.e. report a high confidence when he was right and a low confidence when he was wrong). A standard method to calculate metacognitive efficiency is to compute the meta-d'/d' (Maniscalco \& Lau, 2012). According to the signal detection theory (Green \& Swets, 1988), the d' is a measure of the ability to discriminate the signal from noise (i.e. the correct answer) and is thus a measure of objective (first-order) performance to the task.

\footnotetext{
${ }^{2}$ Note that while the terms metacognitive efficiency and metacognitive (or confidence) bias are widely used in the metacognitive literature, some researchers also use "metacognitive resolution" and "metacognitive calibration" instead.
} 
The meta-d'/d' thus reflects the degree to which a subject can discriminate his judgements as correct or incorrect relative to his performance (Fleming et al., 2014). The d' and Meta-d'/d' were computed using MATLAB code available at http://www.columbia.edu/ bsm2105/type2sdt (Fleming et al., 2014). Before analysis, the confidence level of each participant was divided into four quantiles. Statistical analyses were conducted on log of meta$d ' / d$ ' to correct the non-normality of the ratio scale (see Keene, 1995).

The confidence bias reflects the general tendency of participants to provide low or high confidence ratings (e.g. confidence can be high or low, irrespective of the performance) and is simply calculated as the average confidence rating. Metacognitive efficiency and confidence bias are dissociated so that for instance, participants could have good metacognitive efficiency but report an overall underestimation of the confidence in their performance.

Parametric statistical tests were conducted (paired-sample t-test or repeated measures ANCOVA) to analyze possible differences between the 'threat' and the 'safe' conditions. Nonparametric statistical tests were used when normality assumptions were not satisfied (Wilcoxon test). As the aim of the study was to investigate specifically the effect of induced anxiety on task performance, all reported results included STAI-B scores (trait anxiety) as covariate.

\subsection{Results}

6 out of 29 participants had to be removed before analyses were conducted. We removed participants whose BDI scores were above the cut-off value of 17 for clinical depression $(n=3)$ to control for any confound due to potential psychopathology. One participant was removed because of technical problems with the skin conductance measurement and 2 participants were excluded due to a negative meta-d', precluding calculation of $\log$ (meta-d'/d') (see Fleming et al., 2014). 23 subjects were included in the final (age: 23.69 years $\pm 4.1,14$ females). The mean BDI score was $5.04( \pm 3.9)$ and the mean STAI-B was $43.13( \pm 3.9)$.

Participants reported significantly more anxiety after blocks in the threat condition than after blocks in the safe condition, $(Z=187, p=.002)$. In addition, average skin conductance level was higher in the threat condition, $(Z=40, p=.002)$. No differences in general state anxiety were observed at the end compared to at the start of the experiment, $(Z=117 p=.667)$.

Repeated-measures ANCOVA with Condition ('threat' vs 'safe') as factor and STAI-B as covariate were conducted on the first-order performance measures (accuracy, contrast, RTs, and d') as well as on the second-order performance measures (overall confidence and metacognitive efficiency). 


\section{Perceptual performance}

There was no difference in accuracy (\% correct) between the safe $(71.6 \%)$ and the threat (71.3\%) condition, $(F(1,21)=0.643, p=.432)$, demonstrating the efficiency of the staircase procedure. Participants performed the different conditions at the same difficulty level as the average contrast (in number of dots) did not differ between both conditions, (safe: 5.5, threat: $5.4 ; F(1,21)=0.388, p=.540$ ). Reactions-times were similar in both conditions (safe: $1054 \mathrm{ms,}$ threat, $1042 \mathrm{~ms}$ ). Finally, condition did not affect participants' perceptual discrimination abilities measured by the d' $(F(1,21)=0.125, p=.727)$. STAI-B did not contribute to any of these analyses. Taken together, these results indicate that anxiety induction had no effect on first-order performance.

\section{Metacognitive performance}

Condition had no effect on average confidence $(F(1,21)=2.90, p=.103)$. Participants reported overall the same level of confidence in the safe (3.11) compared to the threat (3.10) condition. STAI-B again did not contribute. However, metacognitive efficiency was lower in the threat compared to the safe condition $\left(F(1,21)=7.87, p=.011, \eta_{p}{ }^{2}=0.273\right)$ (Figure $2 \mathrm{~d}$ ). Under threat, participants showed a reduced ability to accurately recognize and report fluctuations in their own performance. A post-hoc power analysis using G*Power software (Erdfelder, FAul, Buchner, \& Lang, 2009) revealed a power of 0.80 with our sample and effect size. The covariate STAI-B contributed $(p=.027)$ and a significant interaction between condition and STAI-B on metacognitive efficiency was observed $(p=.002)$.

Figure 2

To further explore the nature of this interaction we looked at the correlation between STAI-B and meta-d'/d' within the safe and the threat conditions separately (Figure 3). A negative correlation was observed between STAI-Trait scores and meta-d'/d' in the threat condition $(\mathrm{r}=-0.598, p=.003$, Figure $3 \mathrm{~B})$ but not in the safe condition $(\mathrm{r}=-0.167, p=.447$, Figure 3A). In other words, participants with higher trait-anxiety were more metacognitively affected by the threat of shocks. 
A follow-up analysis was performed with STAI-B removed from the design. The effect of induced anxiety on metacognitive efficiency was still observed when STAI-B was removed $\left(F(1,22)=5.084, p=.034, \eta_{p}{ }^{2}=0.188\right)$. Finally, when compared to an optimal metacognitive score (meta-d'/d' $=1$ ), only metacognitive efficiency in the threat condition was significantly lowered, $(t(22)=-2.424, p=.024, d=0.51)$. Possibly explaining the lack of correlation in the safe condition, participants had maximal performance, using all the available sensory evidence to create their metacognitive judgements (Fleming \& Lau, 2014).

Figure 3

\subsection{Interim discussion}

In Experiment 1, we investigated how induced anxiety that is unrelated for task performance influences participant's metacognitive abilities. For this purpose, we administered electric shocks randomly during a perceptual metacognitive task. Results showed that task-unrelated anxiety had no impact, neither on the first-order task performance nor on the confidence bias. In contrast, metacognitive efficiency decreased. Under threat, participants were less able to correctly identify correct from incorrect performance. The absence of impact of induced anxiety on first-order task performance but well on metacognitive efficiency is consistent with the findings of previous studies (Massoni, 2014; Reyes et al., 2015). This dissociation seems to confirm that anxiety induction has more impact on high-order cognitive processes because the induced emotional load drains some resources that are needed to perform the task correctly (cf. Processing Efficiency Theory, Eysenck et al., 2007). Furthermore, the deterioration of participants' metacognitive efficiency found in the threat condition is in line with our hypothesis of task-relatedness as an important factor determining the direction of the relation between anxiety and cognition. As expected, the unavoidability and uncontrollability of the threat (because induced in a random way) resulted in a deterioration of metacognitive performance. To unequivocally demonstrate that task-relatedness of induced anxiety drives the direction of the relation between anxiety and cognition, also the reverse relation should be demonstrated. If participants believe that their performance can effectively reduce the amount 
of induced anxiety, an improvement in metacognitive performance can be expected. This is exactly what we tested in the following experiment. The task design was identical to the first experiment. Participants again performed the perceptual judgement task and again received electric shock as randomly as in Experiment 1. Importantly however, this time participants were told that they would be more at risk to receive electric shocks if they failed to judge their own performance correctly.

\section{Experiment 2}

\subsection{Method}

\subsubsection{Participants}

Twenty-four right-handed participants were recruited from the Université Libre de Bruxelles and were paid $10 € /$ hour for their participation. All participants gave written informed consent. The study was approved by the Erasmus-ULB Faculty Ethics Committee (P2018/346). Past or current psychiatric and/or neurologic disorder and use of psychoactive medication served as exclusion criteria.

\subsubsection{Anxiety induction}

The electric shocks were calibrated and administrated following the exact same procedure as in Experiment 1. The mean stimulation level was $3.6(\mathrm{SD}=2.3$, average pulse duration; $500 \mu \mathrm{s})$.

\subsubsection{Metacognitive task}

The perceptual metacognitive task was identical as in Experiment 1. On each trial, participants first had to indicate which circle (left or right) contained most dots. Then, participants rated the confidence they had in their choice on a scale from 1 to 6 .

\subsubsection{Procedure}

Apart from instructions, the experimental procedure of Experiment 2 was identical to the procedure of Experiment 1. After completing the BDI-II and STAI A-B, and after the calibration of the intensity of the shocks, participants started the metacognitive task. Different from Experiment 1, participants were informed that the shocks would be given in relation to their metacognitive performance. They were told that there was a higher risk to receive a shock when their confidence judgements did not match the accuracy of their decision. At the end of the experiment, all participants were asked to provide with an informal feedback on the task to ensure they believed the administration of shocks was influenced by their metacognitive performance. None of the participants expressed any doubt with respect to this aspect of the task. 


\subsection{Results}

1 participant was discarded from the final sample because of a BDI score above the cut-off value for clinical depression. 23 subjects were included in the final analysis (age: 22.83 years $\pm 2.9,18$ females). The mean BDI score was $6.48( \pm 5.0)$ and the mean STAI-B was $38.13( \pm$ 10.1).

Both subjective and objective anxiety (skin conductance level) measures were significantly higher in the threat condition compared to the safe condition (respectively $\mathrm{Z}=270, p<.001 ; \mathrm{Z}$ $=239, p=.034)$ meaning that our induction procedure was efficient. Again, participant's STAIA scores were not higher after completing the experiment than before the start $(t(22)=0.262$, $p=.796$ ) meaning that the induced anxiety did not last after the end of the metacognitive task. Repeated-measures ANCOVA with Condition ('threat' vs 'safe') as a factor and STAI-B as covariate were again conducted on the first-order performance measures (accuracy, contrast, RTs, and d') as well as on the second-order performance measures (overall confidence and metacognitive efficiency).

\section{Perceptual performance}

Replicating the findings of Experiment 1, we found no effect of the anxiety induction on first order task performance. There was no difference in accuracy (safe: $70.6 \%$, threat: $71.4 \%$; $F(1,21)=0.005, p=.943$ ), in the contrast level (safe: 6.1 , threat: $5.5 ; F(1,21)=0.274, p=.606$ ) nor in reaction-times (safe: $1095 \mathrm{~ms}$, threat: $1162 \mathrm{~ms}, F(1,21)=1.131, p=.300$, Figure 4A). The STAI-B did not contribute to any of these results (all $p>.100$ ). Finally we found no effect of anxiety on perceptual sensitivity $\left(d^{\prime}\right)^{\prime}(F(1,21)=0.418, p=.525$, Figure 4B). There was a marginal effect of the STAI-B on the $d^{\prime}(F(1,21)=3.733, p=.067)$ with higher d' for increasing trait-anxiety.

Figure 4 


\section{Metacognitive performance}

Different from Experiment 1, an effect of the anxiety induction on the confidence bias $(F(1,21)$ $=11.02, p=.003, \eta_{p}{ }^{2}=0.344$, Figure 4 C) was observed. Participants were overall less confident in the threat condition (4.4) compared to the safe condition (4.2). The covariate STAI-B interacted significantly with the anxiety Condition $\left(F(1,21)=6.42, p=.019, \eta_{p}{ }^{2}=0.234\right)$. Importantly, metacognitive efficiency was higher in the threat compared to the safe condition $\left(F(1,21)=0.018, p=.007, \eta_{p}{ }^{2}=0.300\right.$, Figure 4D). The covariate STAI-B interacted significantly with the anxiety Condition $\left(F(1,21)=6.88, p=.016, \eta_{p}{ }^{2}=0.247\right)$. A positive correlation was found between the STAI-B and the metacognitive efficiency in the safe condition $(\mathrm{r}=0.418, p=.047$, Figure 5A) but not in the threat condition $(\mathrm{r}=.190, p=.386$, Figure 5B). Participants with higher trait anxiety had higher metacognitive efficiency in the safe condition. We will further elaborate on this finding in the general discussion.

Possibly explaining the lack of correlation in the threat condition, participants had maximal performance, using all the available sensory evidence to create their metacognitive judgements $(t(21)=0.038, p=.970, d=0.008)$. In contrast, metacognitive efficiency was significantly lower than 1 in the safe condition $(t(21)=2.226, p=.037, d=.475)$.

Figure 5

\section{Task-related and task-unrelated anxiety}

To directly compare the effect of task-unrelated and task-related anxiety on metacognitive performance, we computed a difference scores between safe and threat conditions for perceptual judgments, for the confidence bias and for metacognitive efficiency. Independent sample T-tests with Experiment as factor were performed on these scores. Perceptual judgments were similar in both experiments $(t(44)=0.466, p=.644)$. For confidence bias, we found a marginal effect of Experiment $(t(44)=1.929, p=.06)$. The difference in confidence between the safe and threat conditions tended to be larger when anxiety was related to the task (Experiment 2). Metacognitive efficiency difference scores differed strongly between experiment 1 and experiment $2(t(44)=3.01, p=.004)$ with a positive difference in Experiment 
1 (mean difference: $0.129 \pm 0.368$ ) and a negative difference (mean difference: $-0.148 \pm 0.245$ ) in Experiment 2.

\section{General discussion}

Different factors influence the relation between anxiety and cognition and could explain why anxiety sometimes improves, sometimes decreases and sometimes has no impact on cognitive performance (Balderston et al., 2017; Grillon, Robinson, Mathur, \& Ernst, 2016; Vytal, Cornwell, Letkiewicz, Arkin, \& Grillon, 2013). In relation with previous studies (Massoni, 2014; Reyes et al., 2015), our findings suggest the importance of at least two factors: the nature of the cognitive processes involved in the task and the task-relatedness of the anxiety. In two experiments, we used the same metacognitive task as Massoni (2014) in which participants had to first perform a low-order perceptual choice and then make a high-order metacognitive judgement about this choice (confidence rating). Following our hypothesis, we predicted that induced anxiety would only influence high-order metacognitive processes without affecting low-order processing. Moreover, we predicted that metacognitive performance would be affected differently depending on whether the anxiety inducing threat was related or not to the metacognitive task. Following the gold standard paradigm to study the effects of anxiety (Robinson et al., 2013), we alternated blocks in which participants received electric shocks (threat blocks) and blocks in which no such shocks were given (safe blocks). In both studies, the shocks were administered randomly, unrelated to the participant's performance. The important and only difference between both experiments was how the electric shocks were perceived to be related to the metacognitive task performance. In experiment 1, participants were informed that shocks would be administered randomly, in a task-unrelated manner. In experiment 2, however, participants were told that the administration of the shocks would be

influenced by their metacognitive performance. Specifically, the instructions were: "you will be more at risk to receive a shock after trial on which you failed to correctly match your confidence with your performance, that is, to give a high confidence when you chose the correct circle and a low confidence when you feel you picked the wrong circle.". Because of the instructions, participants thus believed that the shocks were related to how they judged their performance.

In both experiments, induced anxiety did not change perceptual performance but had a significant impact on metacognitive performance. In agreement with our hypothesis, this impact was in opposite direction for task-related and task-unrelated anxiety. When electric shocks were unrelated for the task (Experiment 1) we found a decreased metacognitive efficiency. 
Participants were less efficient to discriminate their own correct from incorrect performance in the threat compared to the safe condition. In contrast, metacognitive efficiency increased when the electric shocks were believed to be related to the metacognitive judgements (Experiment 2): participants were better at judging their performance in the threat compared to the safe condition. Importantly, there was no objective difference in the manner the shocks were administered between both experiments. The dissociation we found suggests that a change in how participants subjectively perceived the relation between the anxiety and the task is sufficient to influence their performance. Note that we would not have predicted different results if the shocks were objectively related to participant's performance. However, the added value of the present design is to show that the subjective perception of how anxiety relates to the task is sufficient to influence metacognition, independently of its objective relation.

Our results are consistent with previous studies that found changes in metacognitive performance under anxious conditions while perceptual performance remained the same (Massoni, 2014; Reyes et al., 2015). It is possible that high-order metacognitive processes demand more cognitive resources than low-order perceptual choices, leaving no additional resources available to compensate second-order performance. Indeed, the fact that anxiety did not impact perceptual performance but affected metacognitive judgements is consistent with previous studies having shown that anxiety has a stronger impact on high-order cognitive processes (Hadwin et al., 2005; Murray \& Janelle, 2003; Smith et al., 2001). This has been explained by the fact that anxiety consumes attentional resources oriented towards the threat (Eysenck et al., 2007; Murray \& Janelle, 2003). When the task is relatively easy, participants are be able to distribute their attention to both threat and task. In contrast, when the task gets more difficult, additional resources can no longer be allocated, resulting in decreased task performance (Eysenck et al., 2007). The complexity or difficulty of the task (e.g. first-order perceptual vs second-order metacognitive) can change the relation between anxiety on cognition. Note however, that the perceptual discrimination of different numerosities is, on itself, not the simplest task to solve. To make a correct perceptual judgment, participants have to integrate the different features within each circle separately (e.g. size, the density, surface and convex hull occupied by the dots) before making a comparison between both percepts and, finally, make a decision (Gebuis, Cohen Kadosh, \& Gevers, 2016). An alternative explanation could be that the specific alteration of metacognitive processes is due to the fact that introspection induced by the metacognitive task drives attention inward. It has been demonstrated that inward and outward attention systems rely on distinct neuronal networks (e.g. Vanhaudenhuyse et al., 2010). Possibly the network responsible for inward attention is 
more sensitive to anxiety. Effects of anxiety on self-knowledge networks have already been previously studied (Todd, Forstmann, Burgmer, Brooks, \& Galinsky, 2015) showing more egocentric perspective under anxious conditions.

The complexity (first- vs second-order) and/or the nature (driving attention inward or outward) of the tasks seem to be factors that can at least partially explain the intensity with which task performance is affected by the presence of anxiety. However, while future studies should aim at exploring directly the contribution of these factors, they do not seem to influence the direction (negative or positive) of this influence. Instead, the present study shows that another factor, task-relatedness of anxiety, could play this role. Perceived task-unrelated anxiety decreased metacognitive performance (Experiment 1) while task-related anxiety increased metacognitive performance (Experiment 2).

The observation of improved metacognitive performance with perceived task-related induced anxiety conceptually replicates the findings of Massoni (2014). Similar to our second experiment, the anxiety inducing threat of financial loss was directly related to the accuracy of the confidence judgements. In such task-related situation, the attentional resources focused on both the threat and the task, highly overlap, which may prevent the distracting effect of anxiety. Moreover, in this case, improvements of performance are causally related to a reduction of the threat, which adds a motivational incentive to perform well. This is consistent with studies that showed that anxiety improves perceptual processes when threat stimuli served as target for the task (i.e. are task-related) (Pourtois et al., 2013; Robinson et al., 2013). In that situation, the attention captured by this salient emotional stimulus boost its processing thus resulting in improved performance. In contrast, when anxiety is task-unrelated, as in the first experiment, the threat is unpredictable and unavoidable. In this case, the attentional resources oriented towards the threat are distracting, thus resulting in decreased of performance. Together, these results suggest that task-relatedness of anxiety is an important factor that seems to conditionate the direction (positive/negative) of the influence of anxiety on cognition.

It has to be mentioned that the notion of task-relatedness of anxiety is related to the concept of controllability. A large number of animal and human studies have shown that controllability over stressors exerts a strong impact on behavior (e.g. Lucas et al., 2014; Maier \& Watkins, 1998). For instance, a lack of controllability is thought to be a key factor in fear sensitization and the development of anxiety disorders (Maier \& Watkins, 1998). In the present study, it is likely that participants had higher feelings of controllability over the threat in the task-related condition as they believed they could reduce the risk of receiving a shock by improving their performance. Our study therefore suggests that perceived controllability, in the 
absence of objective controllability, is sufficient to influence metacognition. However, the question whether task-relatedness and controllability fully overlap remains to be clarified. Whether a threat is controllable or not should depend on the possibility we have to act on the parameter that can reduce the threat. For instance, imagine a task in which the threat is related to participant's speed (i.e., they receive a shock if they responded slower than $1000 \mathrm{~ms}$ ). In that case, the anxiety induction is task-related and controllable. Participants can voluntarily speed up their responses to avoid the shocks. In the present study, the question is thus whether metacognitive efficiency is under voluntary control or not. Studies have shown that metacognitive performance can be improved by specific training (Carpenter et al., 2019) or with task expectations (Sherman, Seth, Barrett, \& Kanai, 2015). However, no study to our knowledge has found that participants can voluntarily increase their metacognitive efficiency with just their own will. This question thus remains to be investigated in future studies.

In both experiments, we explored the relation between trait-anxiety, as measured by the STAI-B and metacognitive efficiency. Participants demonstrated nearly perfect metacognitive performance (e.g. participants used all available sensory evidence to create their metacognitive judgement) in the safe condition of Experiment 1 and in the threat condition of experiment 2. This possibly explains the absence of correlation between trait anxiety and metacognitive efficiency in these conditions. In experiment 1 , higher levels of trait-anxiety were associated with lower metacognitive efficiency in the threat condition. An intuitively appealing interpretation is that participants with higher trait-anxiety are more metacognitively affected by the threat of shocks. This is consistent with the study of Reyes and colleagues (2015) having found impaired metacognitive efficiency in participants with high disposition to stress. A different picture emerged in Experiment 2. Higher trait-anxiety was now associated with better metacognitive efficiency in the safe condition. This finding was not anticipated, and our interpretation is post-hoc. Anecdotally, some participants reported that they viewed the safe condition as an opportunity to relax, even at the cost of more incorrect metacognitive judgements. It could very well be that participants with higher trait-anxiety "relaxed less" in the safe condition, relatively resulting in a higher focus and better metacognitive efficiency. More research is clearly needed on the relation between trait-anxiety and metacognitive performance.

Our results also showed a dissociation between metacognitive efficiency and the overall confidence (i.e. confidence bias). In Experiment 1, while task-unrelated anxiety decreased metacognitive efficiency, it did not impact the confidence bias. In Experiment 2, participants had increased metacognitive efficiency but reported lower overall confidence with perceived 
task-related anxiety. In this case, it is likely that the lower confidence reflects a more cautious strategy in an attempt to avoid the shocks. However, note that Massoni (2014) did not find any effect of task-related anxiety on confidence bias.

We did not assess other emotional affects that participants could have experienced during the manipulation. We thus cannot rule out the hypothesis that other emotions such as sadness or anger had an influence on metacognitive performance. Note also that while we used the term anxiety in our study, Massoni (2014) measured the negative emotion they induced in terms of "worry". Even if these two terms are very close constructs, the extent to which they totally overlap remains to be investigated. Finally, while threat of shocks is commonly used as anxiety induction procedures, they represent a relatively extreme case of anxious stimuli. Future studies should aim at replicating the present findings in more ecological situations, as did Massoni (2014) with monetary losses for instance.

In conclusion, the present study underlined the importance of at least two factors in the relation between anxiety and cognition: the complexity and/or nature of cognitive processes involved in the task and perceived task-relatedness of anxiety. Together with previous studies, our findings suggest that these two factors could play different roles in this relation and could interact together. The demands of the tasks seem to influence the intensity of the influence of anxiety, with higher-order cognitive tasks being more strongly impacted. In contrast, taskrelatedness of anxiety seem to conditionate the direction of this influence, with an improved performance in case of related anxiety and decreased performance in case of unrelated anxiety.

\section{Acknowledgements}

We thank Stephen Fleming for sharing his code helping us to design our experiment. We also thank Netty Pastour and Jean-Baptiste Immers for their valuable help during the testings.

\section{Funding}

This paper was supported by a Mini-Arc grant from the Université Libre de Bruxelles (A.R. 5/7/96 - M.B. 27/8/96).

\section{Data availability statement}

Data are available at https://osf.io/p2697 


\section{References}

Anderson, A. K., \& Phelps, E. A. (2001). Lesions of the human amygdala impair enhanced perception of emotionally salient events. Nature, 411(6835), 305-309. https://doi.org/10.1038/35077083

Ashcraft, M., \& Kirk, E. (2001). The Relationships Among Working Memory, Math Anxiety, and Performance. Journal of Experimental Psychology. General, 130(2), 224-237. https://doi.org/10.1037//0096-3445.130.2.224

Balderston, N. L., Hale, E., Hsiung, A., Torrisi, S., Holroyd, T., Carver, F. W., ... Grillon, C. (2017). Threat of shock increases excitability and connectivity of the intraparietal sulcus. ELife, 6. https://doi.org/10.7554/eLife.23608

Beck, A., Ward, C., Mendelson, M., Mock, J., \& Erbaugh, J. (1961). An inventory for measuring depression. Archives of General Psychiatry, 4(6), 561-571. Retrieved from http://dx.doi.org/10.1001/archpsyc.1961.01710120031004

Bourque, P., \& Beaudette, D. (1982). Etude psychométrique du questionnaire de dépression de Beck auprès d'un échantillon d'étudiants universitaires francophones. Canadian Journal of Behavioural Science, 14(3), 211-218. https://doi.org/10.1037/h0081254

Brainard, D. (1997). The Psychophysics Toolbox. Spatial Vision, 10(4), 433-436. https://doi.org/10.1163/156856897X00357

Braithwaite, J., Watson, D., Robert, J., \& Mickey, R. (2013). A Guide for Analysing Electrodermal Activity (EDA) \& Skin Conductance Responses (SCRs) for Psychological Experiments. .... https://doi.org/10.1017.S0142716405050034

Carpenter, J., Sherman, M. T., Kievit, R. A., Seth, A. K., Lau, H., \& Fleming, S. M. (2019). Domain-general enhancements of metacognitive ability through adaptive training. Journal of Experimental Psychology: General, 148(1), 51-64. https://doi.org/10.1037/xge0000505

Caspar, E. A., Christensen, J. F., Cleeremans, A., \& Haggard, P. (2016). Coercion Changes the Sense of Agency in the Human Brain. Current Biology, 26(5), 585-592. https://doi.org/10.1016/j.cub.2015.12.067

Davis, M., Walker, D. L., Miles, L., \& Grillon, C. (2010, January 19). Phasic vs sustained fear in rats and humans: Role of the extended amygdala in fear vs anxiety. Neuropsychopharmacology. Nature Publishing Group. 
https://doi.org/10.1038/npp.2009.109

Erdfelder, E., FAul, F., Buchner, A., \& Lang, A. G. (2009). Statistical power analyses using G*Power 3.1: Tests for correlation and regression analyses. Behavior Research Methods, 41(4), 1149-1160. https://doi.org/10.3758/BRM.41.4.1149

Eysenck, M. W., Derakshan, N., Santos, R., \& Calvo, M. G. (2007). Anxiety and cognitive performance: Attentional control theory. Emotion, 7(2), 336-353. https://doi.org/10.1037/1528-3542.7.2.336

Flavell, J. H. (1979). Metacognition and cognitive monitoring: A new area of cognitivedevelopmental inquiry. American Psychologist, 34(10), 906-911. https://doi.org/10.1037/0003-066X.34.10.906

Fleming, S. M., \& Dolan, R. J. (2012). The neural basis of metacognitive ability. The Cognitive Neuroscience of Metacognition (Vol. 9783642451). https://doi.org/10.1098/rstb.2011.0417

Fleming, S. M., Ryu, J., Golfinos, J. G., \& Blackmon, K. E. (2014). Domain-specific impairment in metacognitive accuracy following anterior prefrontal lesions. Brain, 137(10), 2811-2822. https://doi.org/10.1093/brain/awu221

Gebuis, T., Cohen Kadosh, R., \& Gevers, W. (2016). Sensory-integration system rather than approximate number system underlies numerosity processing: A critical review. Acta Psychologica, 171, 17-35. https://doi.org/10.1016/j.actpsy.2016.09.003

Grant, D. M., \& White, E. J. (2016). Influence of Anxiety on Cognitive Control Processes, 1(September), 1-27. https://doi.org/10.1093/acrefore/9780190236557.013.74

Green, D. M., \& Swets, J. A. (1988). Signal detection theory and psychophysics. Peninsula Pub.

Grillon, C., Robinson, O. J., Mathur, A., \& Ernst, M. (2016). Effect of attention control on sustained attention during induced anxiety. Cognition and Emotion, 30(4), 700-712. https://doi.org/10.1080/02699931.2015.1024614

Hadwin, J. A., Brogan, J., \& Stevenson, J. (2005). State anxiety and working memory in children: A test of processing efficiency theory. Educational Psychology, 25(4), 379393. https://doi.org/10.1080/01443410500041607

Iordan, A. D., Dolcos, S., \& Dolcos, F. (2013). Neural signatures of the response to emotional distraction: A review of evidence from brain imaging investigations. Frontiers in Human Neuroscience, 7(JUN), 200. https://doi.org/10.3389/fnhum.2013.00200

Keene, O. N. (1995). the Log Transformation Is Special, 14(April 1994), 811-819.

Lucas, M., Ilin, Y., Anunu, R., Kehat, O., Xu, L., Desmedt, A., \& Richter-Levin, G. (2014). 
Long-term effects of controllability or the lack of it on coping abilities and stress resilience in the rat. Stress, 17(5), 423-430. https://doi.org/10.3109/10253890.2014.930430

Maier, S. F., \& Watkins, L. R. (1998). Stressor Controllability, Anxiety, and Serotonin. Cognitive Therapy and Research, 22(6), 595-613. https://doi.org/10.1023/A:1018794104325

Maniscalco, B., \& Lau, H. (2012). A signal detection theoretic approach for estimating metacognitive sensitivity from confidence ratings. Consciousness and Cognition, 21(1), 422-430. https://doi.org/10.1016/j.concog.2011.09.021

Massoni, S. (2014). Emotion as a boost to metacognition: How worry enhances the quality of confidence. Consciousness and Cognition, 29, 189-198. https://doi.org/10.1016/j.concog.2014.08.006

Moser, J. S., Moran, T. P., Schroder, H. S., Donnellan, M. B., \& Yeung, N. (2013). On the relationship between anxiety and error monitoring: A meta-analysis and conceptual framework. Frontiers in Human Neuroscience, 7(AUG), 466. https://doi.org/10.3389/fnhum.2013.00466

Murray, N. P., \& Janelle, C. M. (2003). Anxiety and Performance: A Visual Search Examination of the Processing Efficiency Theory. Journal of Sport and Exercise Psychology, 25(2), 171-187. https://doi.org/10.1123/jsep.25.2.171

Palmer, E. C., David, A. S., \& Fleming, S. M. (2014). Effects of age on metacognitive efficiency. Consciousness and Cognition, 28(1), 151-160. https://doi.org/10.1016/j.concog.2014.06.007

Paradiso, S. (1998). The Emotional Brain: The Mysterious Underpinnings of Emotional Life. American Journal of Psychiatry, 155(4), 570-570. https://doi.org/10.1176/ajp.155.4.570

Pourtois, G., Schettino, A., \& Vuilleumier, P. (2013, March 1). Brain mechanisms for emotional influences on perception and attention: What is magic and what is not. Biological Psychology. Elsevier. https://doi.org/10.1016/j.biopsycho.2012.02.007

Reyes, G., Silva, J. R., Jaramillo, K., Rehbein, L., \& Sackur, J. (2015). Self-Knowledge DimOut: Stress Impairs Metacognitive Accuracy. PLoS ONE, 10(8), 1-10. https://doi.org/10.1371/journal.pone.0132320

Robinson, O. J., Vytal, K., Cornwell, B. R., \& Grillon, C. (2013). The impact of anxiety upon cognition: perspectives from human threat of shock studies. Frontiers in Human Neuroscience, 7(May), 1-21. https://doi.org/10.3389/fnhum.2013.00203

Schmitz, A., \& Grillon, C. (2012). Assessing fear and anxiety in humans using the threat of 
predictable and unpredictable aversive events (the NPU-threat test). Nature Protocols, 7(3), 527-532. https://doi.org/10.1038/nprot.2012.001

Sherman, M. T., Seth, A. K., Barrett, A. B., \& Kanai, R. (2015). Prior expectations facilitate metacognition for perceptual decision. Consciousness and Cognition, 35, 53-65. https://doi.org/10.1016/j.concog.2015.04.015

Siman-Tov, T., Papo, D., Gadoth, N., Schonberg, T., Mendelsohn, A., Perry, D., \& Hendler, T. (2009). Mind your left: Spatial bias in subcortical fear processing. Journal of Cognitive Neuroscience, 21(9), 1782-1789. https://doi.org/10.1162/jocn.2009.21120

Smith, N. C., Bellamy, M., Collins, D. J., \& Newell, D. (2001). A test of processing efficiency theory in a team sport context. Journal of Sports Sciences, 19(5), 321-332. https://doi.org/10.1080/02640410152006090

Spielberger, C. D., Gorsush, R. L., Lushene, R., Vagg, P. R., \& Jacobs, G. A. (1983). Manual for the State-Trait Anxiety Inventory. Palo Alto, CA: Consulting Psychologists Press.

Todd, A. R., Forstmann, M., Burgmer, P., Brooks, A. W., \& Galinsky, A. D. (2015). Anxious and egocentric: How specific emotions influence perspective taking. Journal of Experimental Psychology: General, 144(2), 374-391. https://doi.org/10.1037/xge0000048

Torrisi, S., Robinson, O., O’Connell, K., Davis, A., Balderston, N., Ernst, M., \& Grillon, C. (2016). The neural basis of improved cognitive performance by threat of shock. Social Cognitive and Affective Neuroscience, 11(11), 1677-1686. https://doi.org/10.1093/scan/nsw088

Vanhaudenhuyse, A., Demertzi, A., Schabus, M., Noirhomme, Q., Bredart, S., Boly, M., ... Laureys, S. (2010). Vanhaudenhuyse et al. - 2010 - Two Distinct Neuronal Networks Mediate the Awareness of Environment and of Self.pdf, 570-578.

Vytal, K. E., Cornwell, B. R., Letkiewicz, A. M., Arkin, N. E., \& Grillon, C. (2013). The complex interaction between anxiety and cognition: Insight from spatial and verbal working memory. Frontiers in Human Neuroscience, 7(MAR), 1-11. https://doi.org/10.3389/fnhum.2013.00093

Wood, J., Mathews, A., \& Dalgleish, T. (2001). Anxiety and Cognitive Inhibition. Emotion, 1(2), 166-181. https://doi.org/10.1037/1528-3542.1.2.166 
Figure 1. Experimental design (Experiment 1 and 2). On each trial, participants had to indicate which circle (left or right) contained most dots. Dots stayed on screen during $700 \mathrm{~ms}$. After the trial, the sentence in French "Lequel contient le plus de points?" ("which one contains most dots?") was presented below the circles. Then, participants had to rate their confidence ("Confiance?") in their choice, on a scale from 1 to 6.

Figure 2. Behavioral and metacognitive measures in 'safe' and 'threat' conditions in Experiment 1. (A) Reaction-Times (ms) did not differ between 'safe' and 'threat' conditions. (B) d' measure, estimated based on Hit rate and False alarm rate, reflects stimulus sensitivity and did not differ between both conditions. (C) Mean confidence ratings did not differ between both conditions. (D) Metacognitive accuracy $[\log ($ meta-d'/d')] in 'safe' and 'threat' conditions. Metacognitive accuracy was significantly lowered in 'threat' condition. In A-D, error bars reflect standard errors. ${ }^{*} \mathrm{p}<0.05$.

Figure 3. Correlations between trait anxiety (STAI-B scores) and meta-d'/d' (metacognitive efficiency in Experiment 1. (A) In the safe condition, trait anxiety and metacognitive efficiency were not correlated $(\mathrm{r}=-0.167, p=.447)$. (B) In the threat condition, we found a negative correlation between trait anxiety and metacognitive efficiency $(\mathrm{r}=-0.598, p=.003)$, meaning that participants with higher trait anxiety were more metacognitively affected by the threat of shocks. The regression lines are indicated with $95 \%$ confidence interval.

Figure 4. Behavioral and metacognitive measures in 'safe' and 'threat' conditions in Experiment 2. (A) Reaction-Times (ms) did not differ between 'safe' and 'threat' conditions. (B) d' measure did not differ between both conditions. (C) Confidence bias (overall confidence ratings) were lower in the threat condition compared to the safe condition. (D) Metacognitive accuracy $[\log ($ meta-d'/d')] in 'safe' and 'threat' conditions. Conversely to Experiment1, Metacognitive accuracy was significantly higher in 'threat' condition. In A-D, error bars reflect standard errors. ${ }^{* *} \mathrm{p}<0.01$.

Figure 5. Correlations between trait anxiety (STAI-B scores) and meta-d'/d' (metacognitive efficiency in Experiment 2. (A) trait anxiety and metacognitive efficiency were positively correlated in the safe condition $(\mathrm{r}=0.418, p=.047)$, meaning that participants with higher trait anxiety had a better metacognitive efficiency. (B) In the threat condition, we found no correlation between trait anxiety and metacognitive efficiency $(r=.190, p=.386)$. The regression lines are indicated with $95 \%$ confidence interval. 

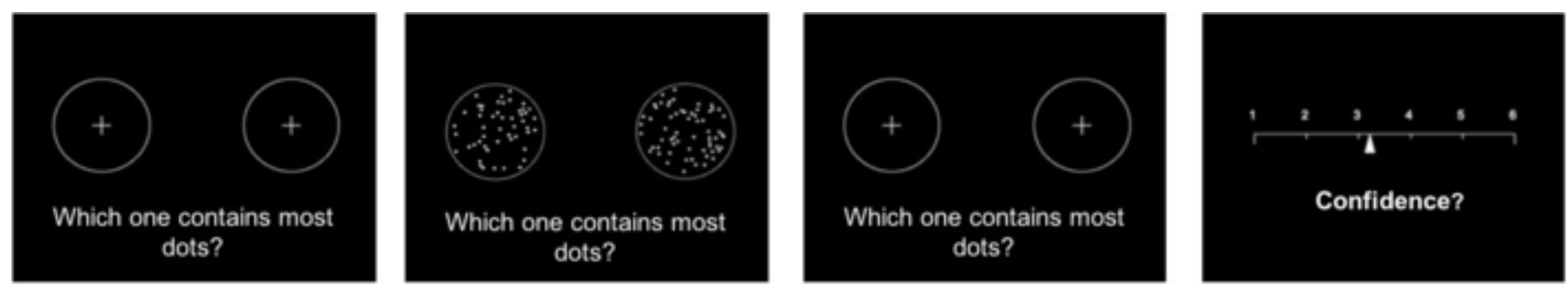

\section{$1000 \mathrm{~ms}$}

$700 \mathrm{~ms}$

Until response

$\leftarrow$ or $\rightarrow$

\section{$3500 \mathrm{~ms}$}


A.

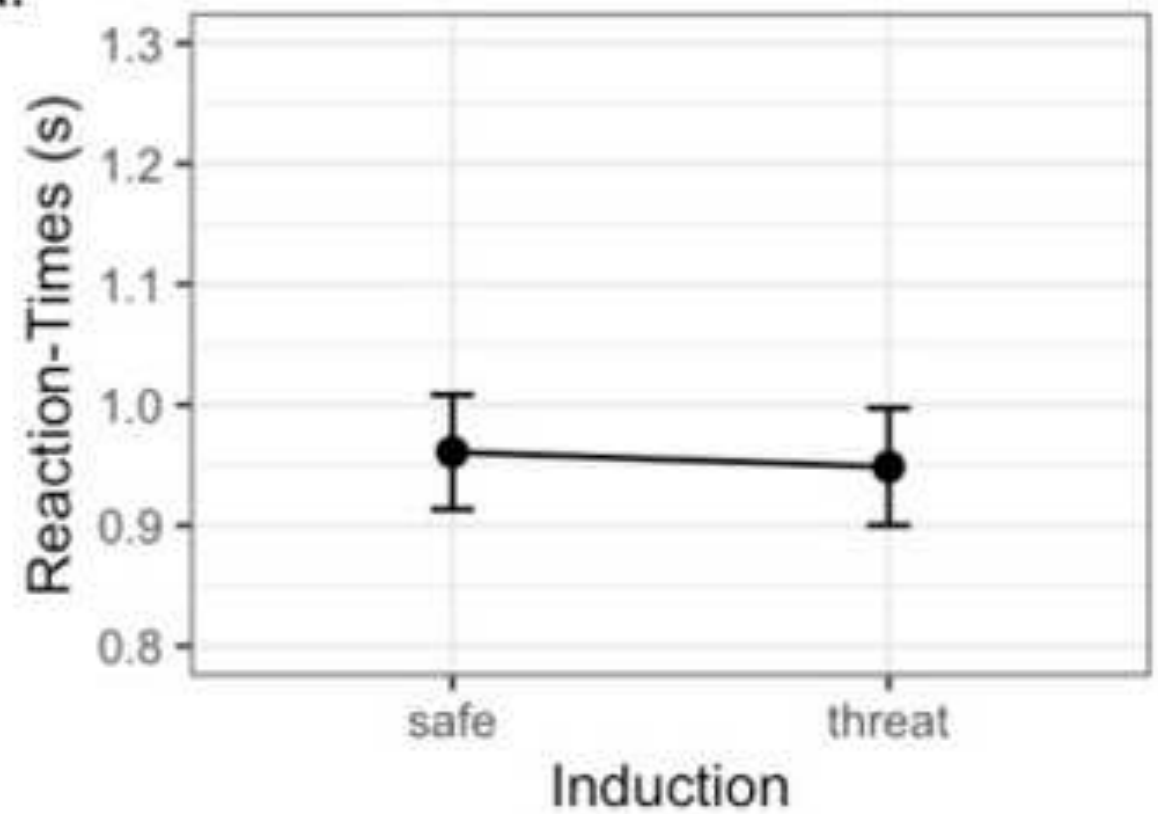

C.

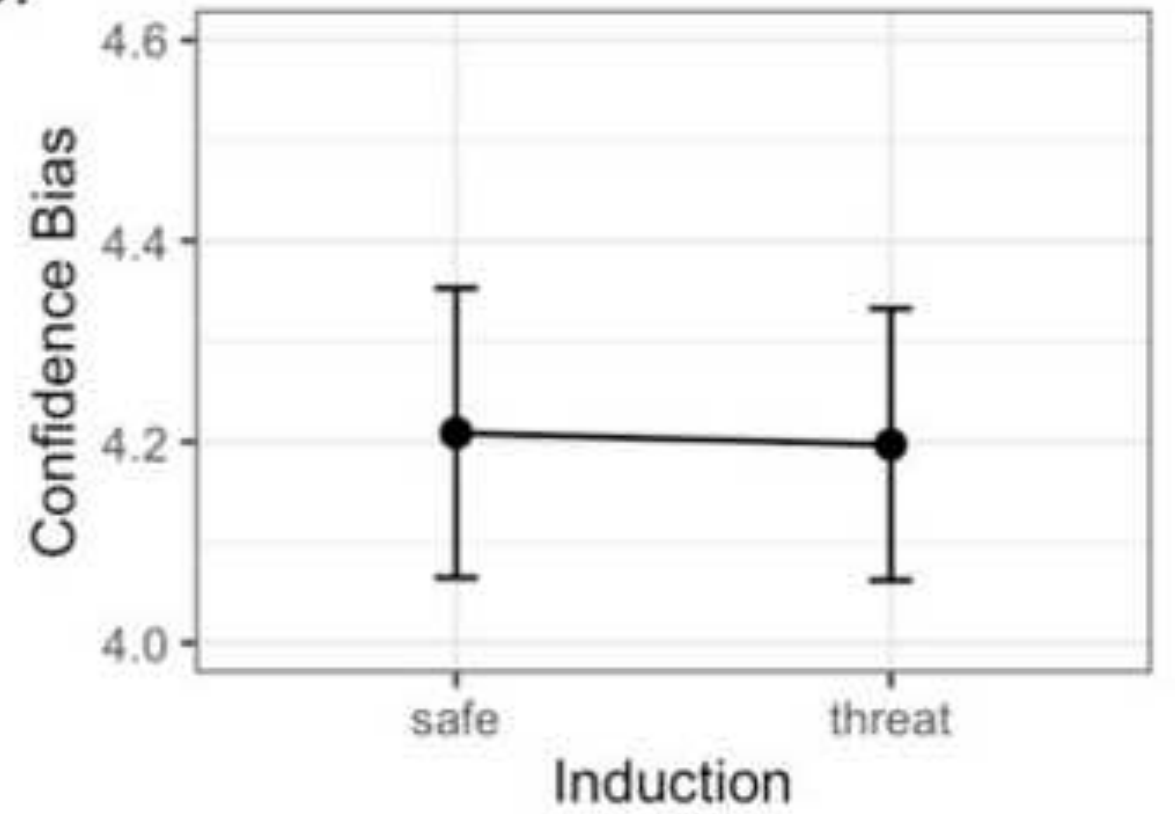

B.

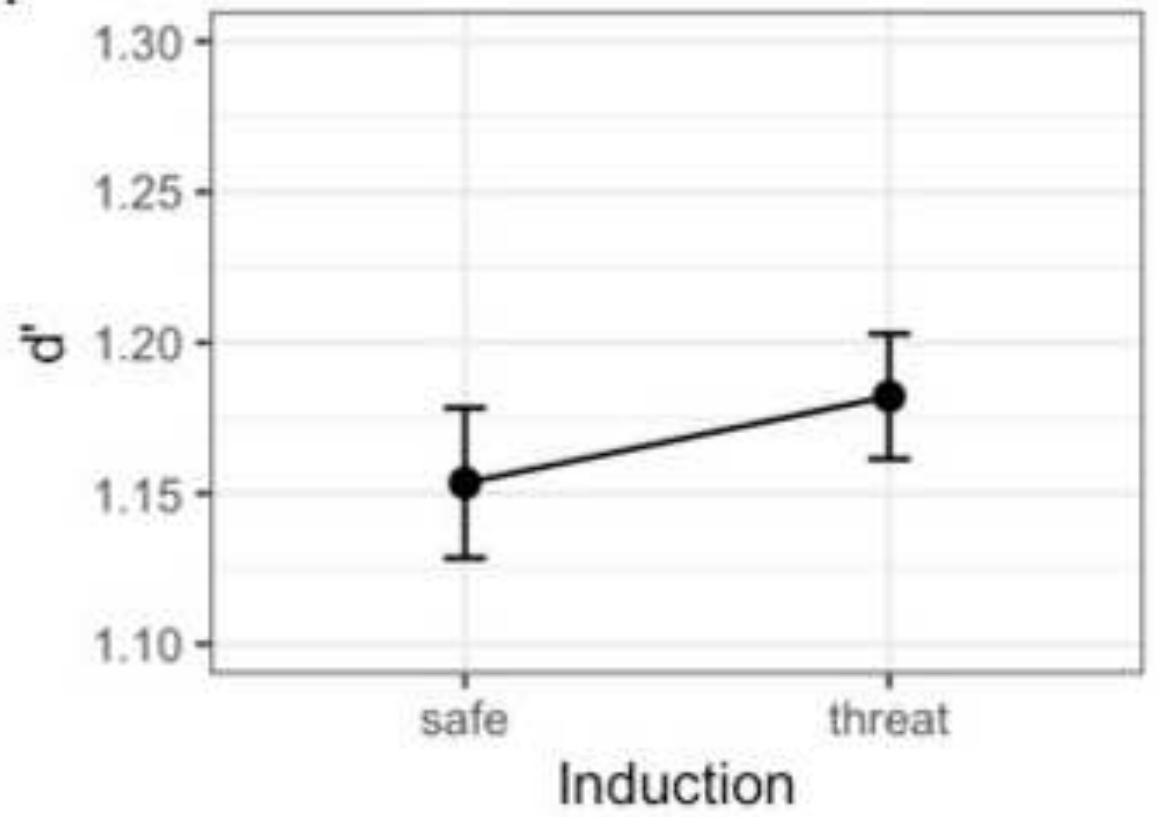

D.

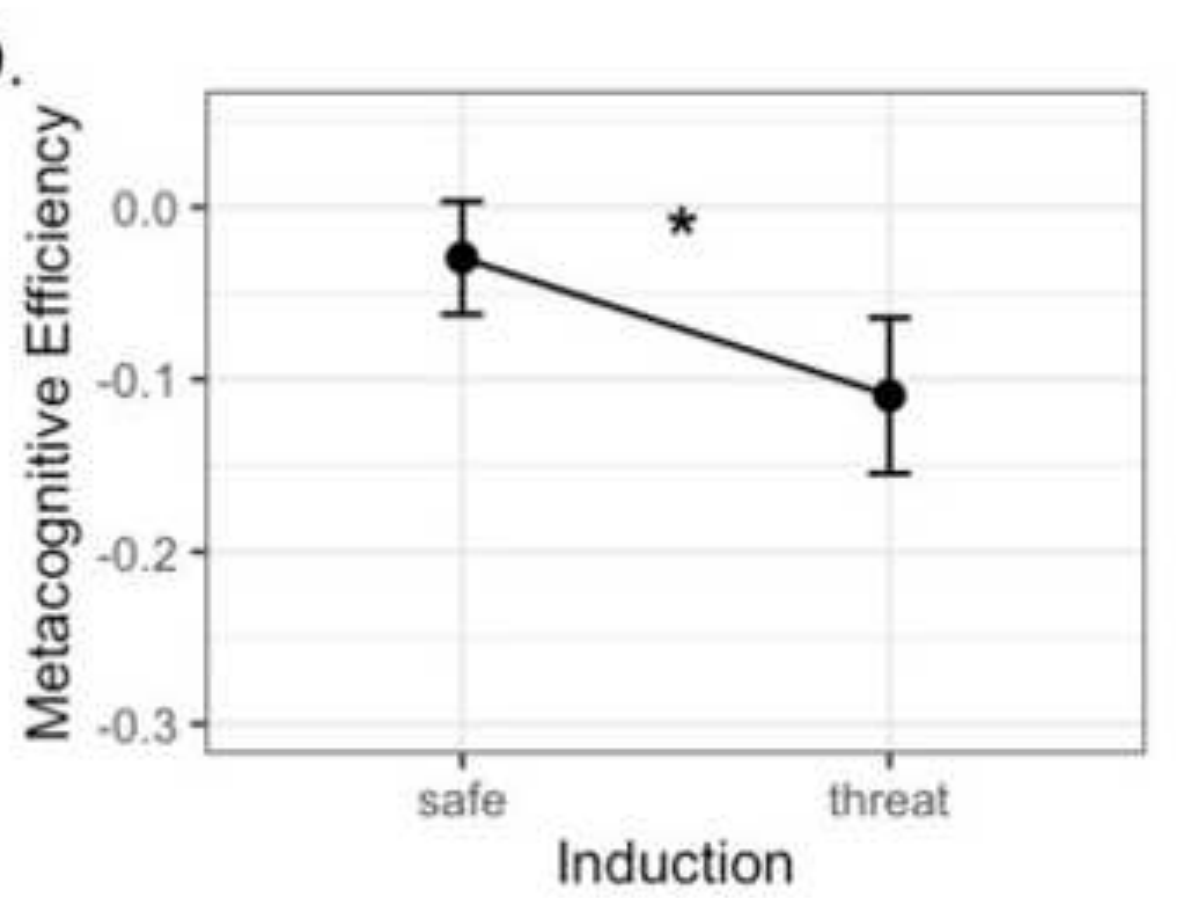




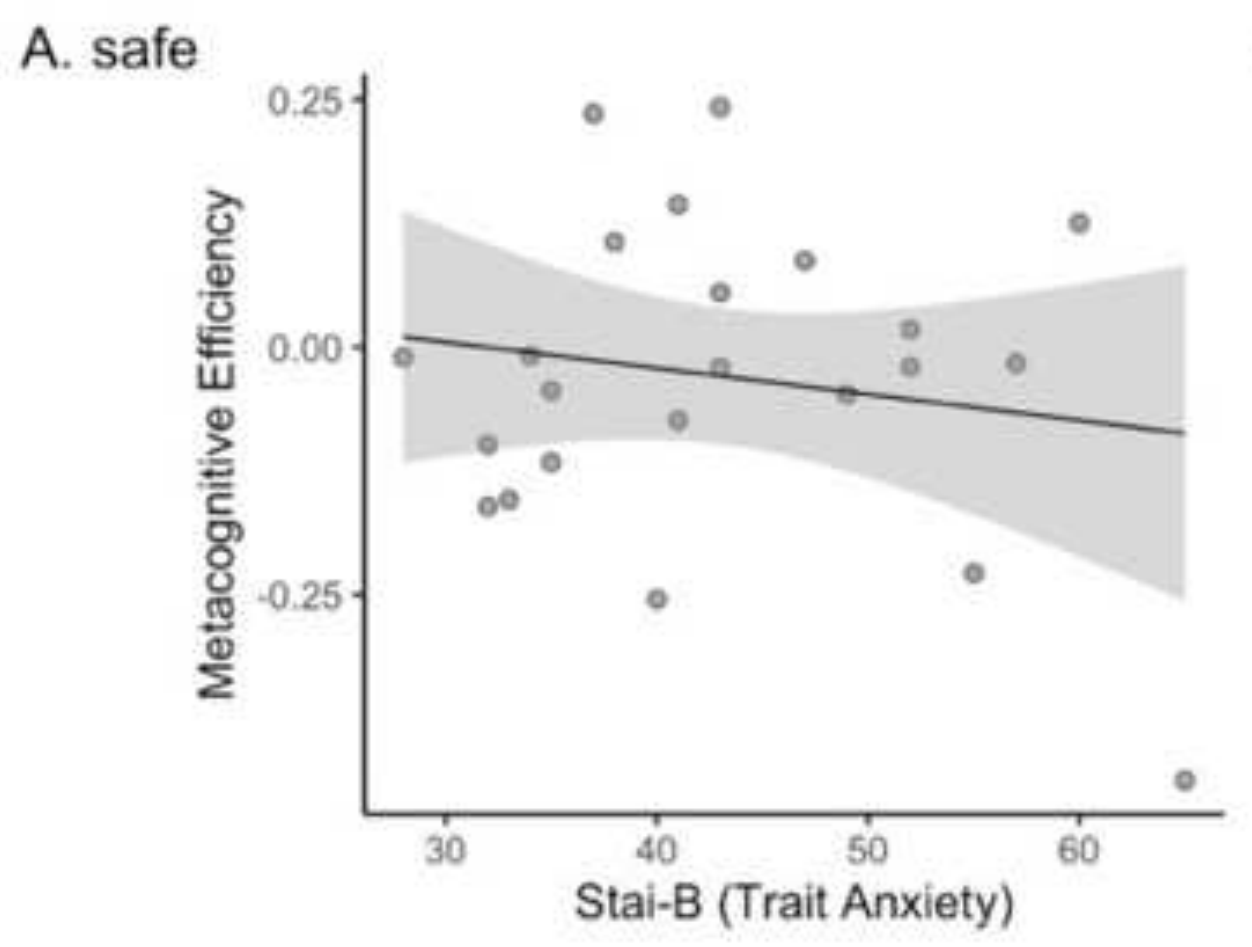

B. threat

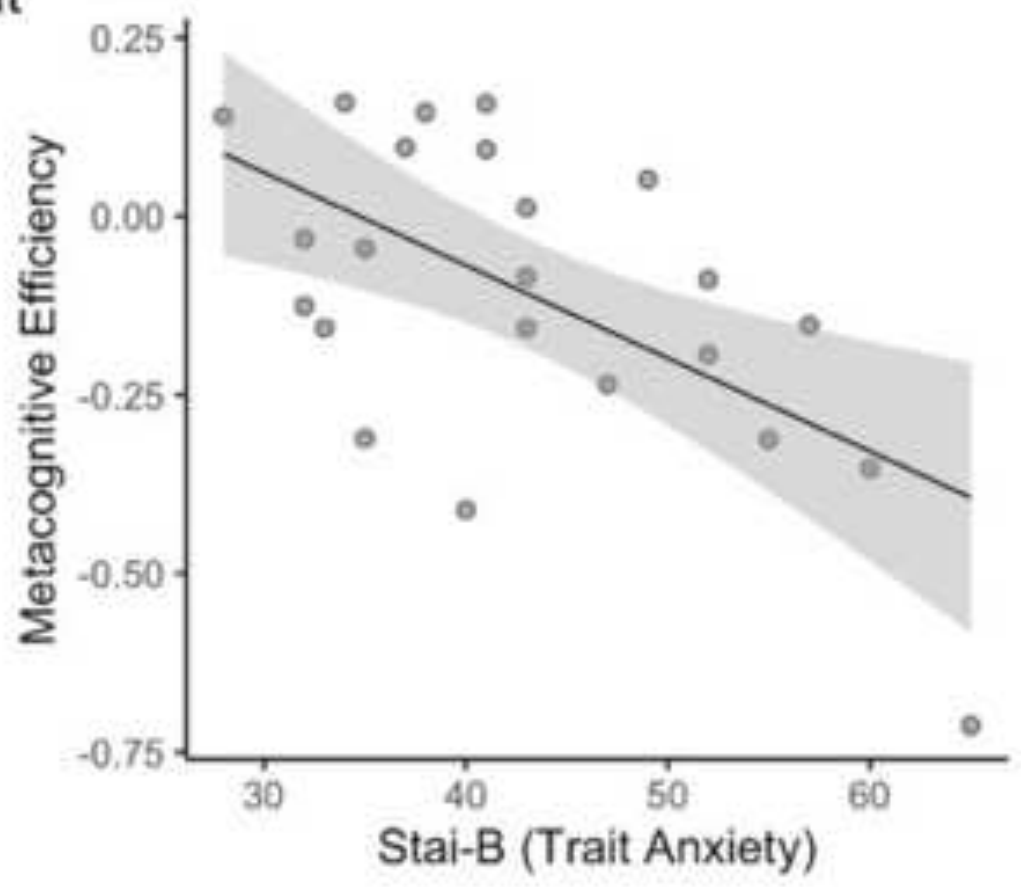


A.

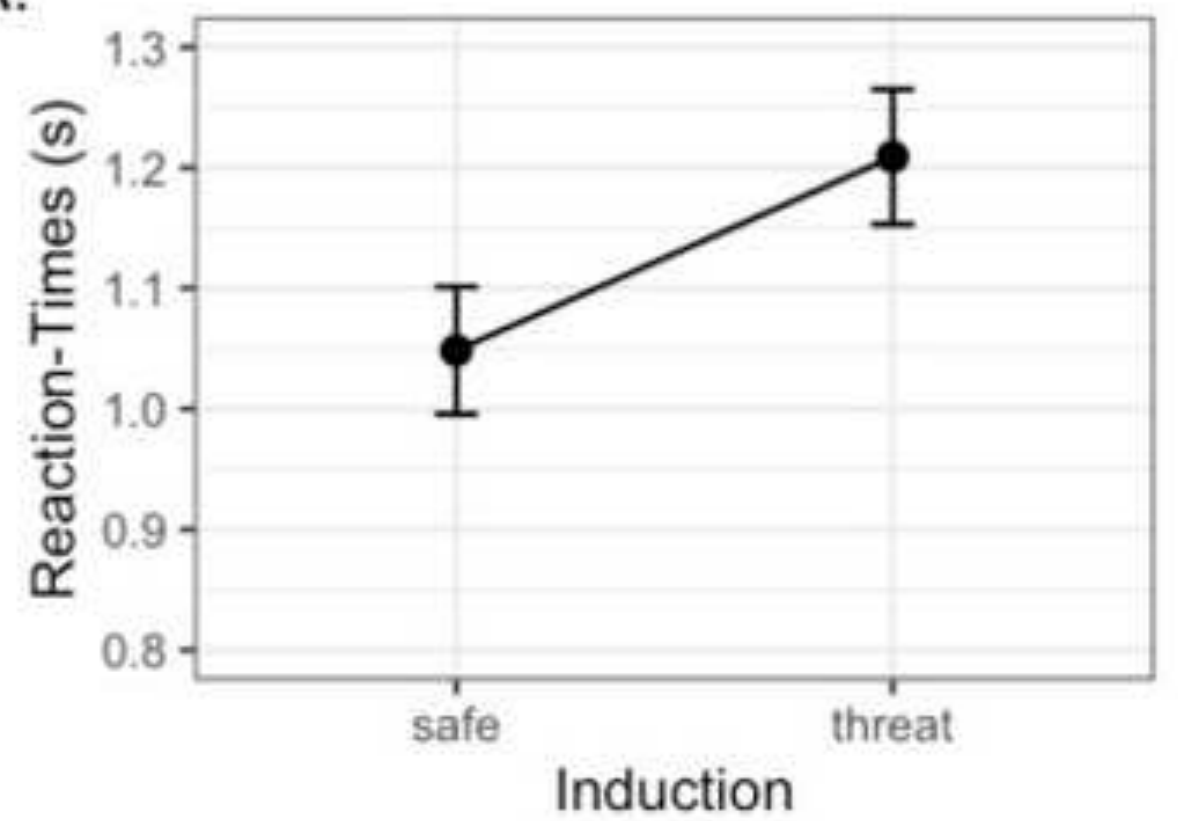

C.

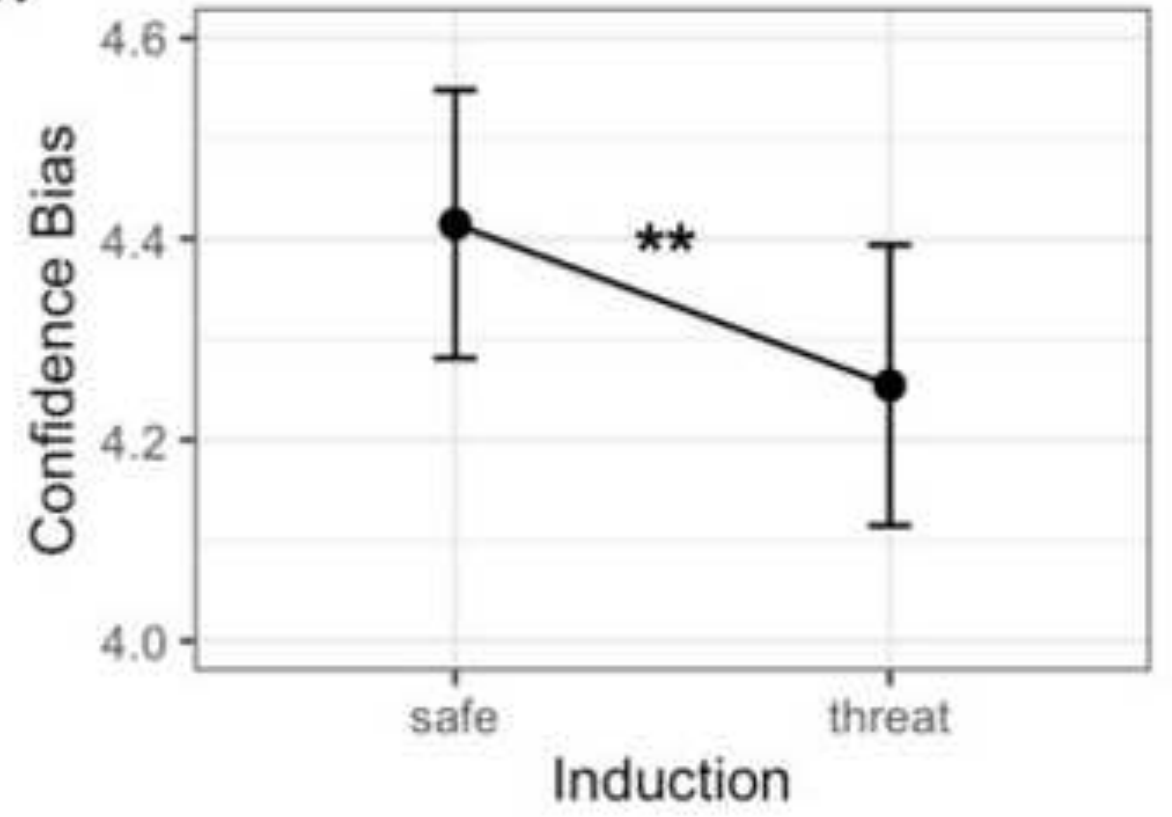

B.

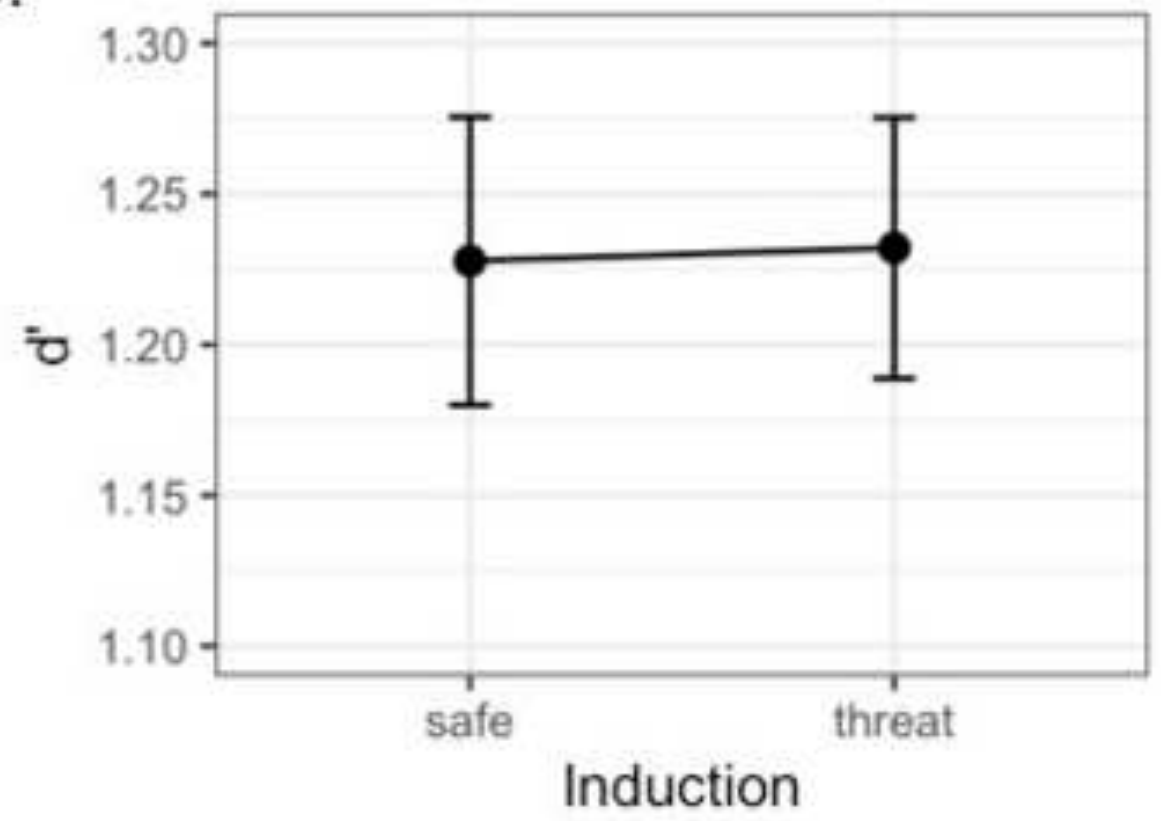

D.

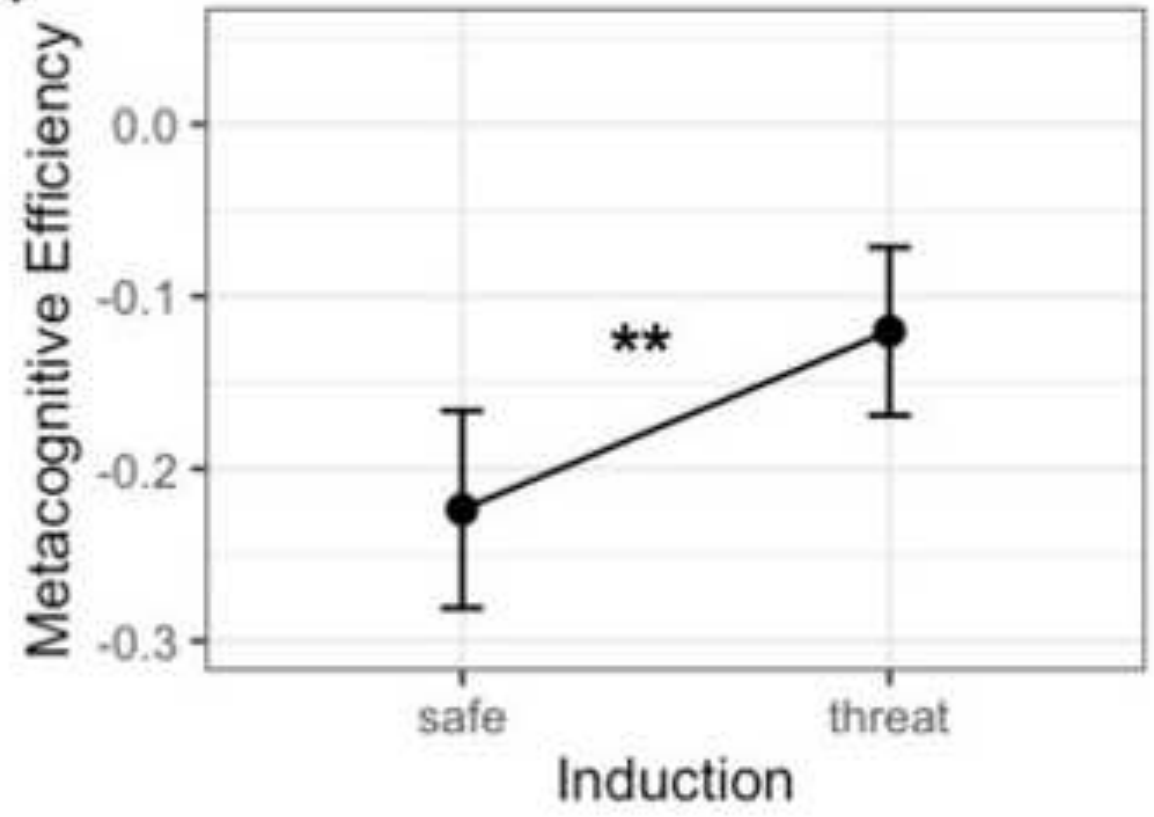


A. safe

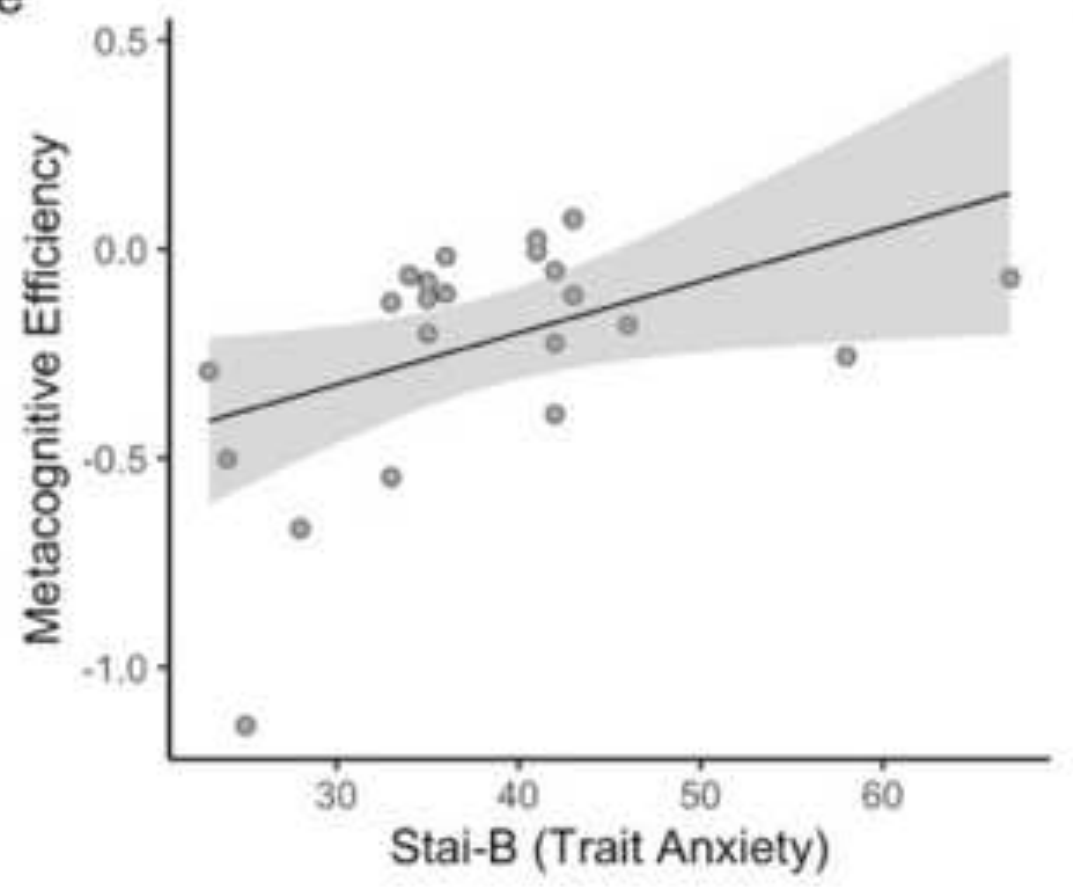

B. threat

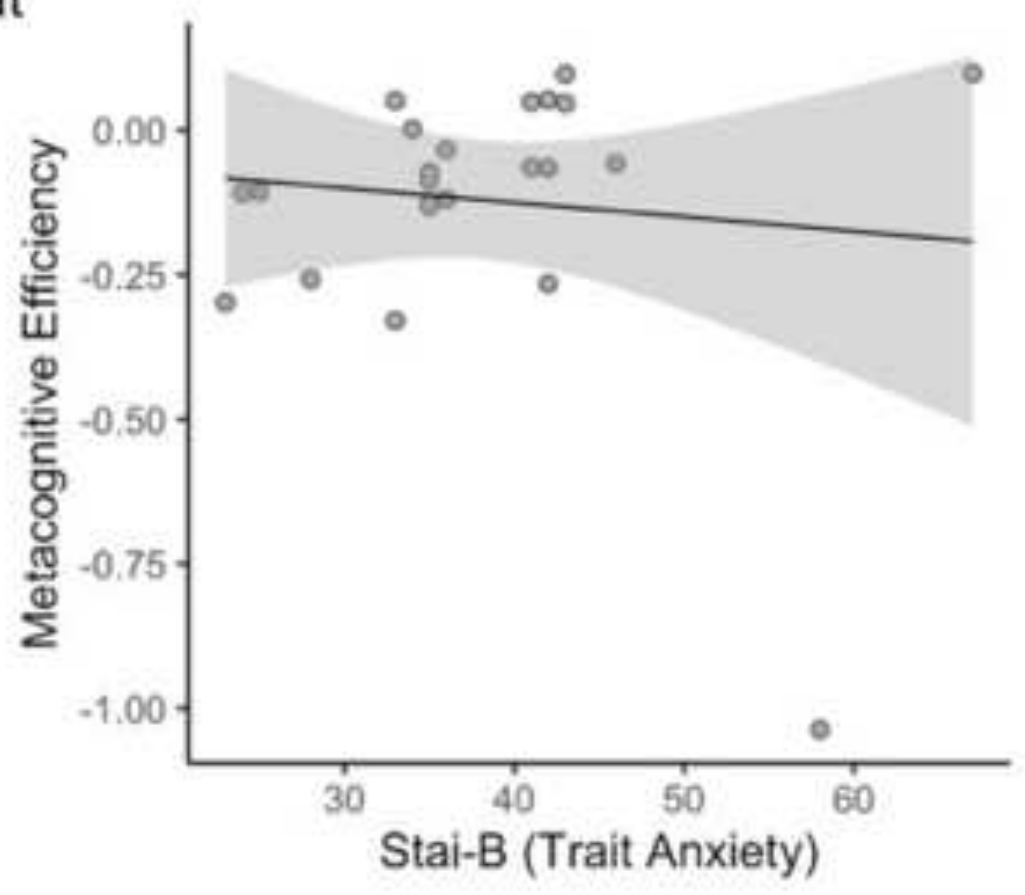




\section{Author Statement}

Catherine Culot: Conceptualization, Methodology, Software, Formal Analysis, Investigation, Writing - Original Draft. Gaia Corlazzoli: Conceptualization, Investigation, Writing - Review and Editing. Carole Fantini-Hauwel: Conceptualization, Writing - Review and Editing. Wim Gevers: Conceptualization, Writing - Review and Editing, Supervision, Project administration. 